\title{
Supporting Effective Monitoring and Knowledge Building in Online Collaborative Learning Systems
}

\author{
Santi Caballé ${ }^{1}$, Angel A. Juan ${ }^{1}$, and Fatos Xhafa ${ }^{2}$ \\ ${ }^{1}$ Open University of Catalonia, Dept of Computer Science \\ Rambla Poblenou, 156. 08018 Barcelona, Spain \\ \{scaballe, ajuanp\}@uoc.edu \\ ${ }^{2}$ Polytechnic University of Catalonia, Dept. of Languages and Informatics Systems \\ Jordi Girona, 1-3. 08034 Barcelona \\ fatos@1si.upc.edu
}

\begin{abstract}
This paper aims to report on an experience of using an innovative groupware tool to support real, collaborative learning. We base the success of on-line collaborative learning on extracting relevant knowledge from interaction data analysis in order to provide learners and instructors with efficient awareness, feedback, and monitoring as regards individual and group performance and collaboration. Monitoring is especially important for online instructors since they can use this valuable provision of information as a meta cognitive tool for regulating the collaborative learning process more conveniently and provide adequate support when needed. In addition, learning and knowledge building may be greatly enhanced by presenting selected knowledge to learners as for their particular skills exhibited during interaction, such as the impact and effectiveness of their contributions. Indeed, by letting learners be aware of both their own and others' progress in the process of knowledge building may promote learners' participation and boost group performance. The ultimate goal of this paper is to provide a model to achieve a more effective support and assessment of the collaborative process while enhancing and improving the learning experience. To validate this study, a real online learning environment is employed to support asynchronous collaborative activities.
\end{abstract}

Keywords: Collaborative Learning, Groupware, Knowledge Discovery.

\section{Introduction}

A relevant research topic in Computer Supported Collaborative Learning (CSCL) is to explore the importance of efficient management of event information generated from group activity in collaborative learning practices for its further use in extracting and providing knowledge on interaction behavior [1]. This view is especially notorious in the current shifting from a traditional educational paradigm (centered in the figure of a masterful instructor) to an emergent educational paradigm which considers students as active and central actors in their learning process. In the Age of the Knowledge Society, students learn, with the help of instructors, technology and other students,

Caballé, S., Juan, Á., Xhafa, F. Supporting effective monitoring and knowledge building in online collaborative learning systems. A: World Summit on the Knowledge Society. "Emerging technologies and information systems for the knowledge society: First World Summit on the Knowledge Society, WSKS 2008: Athens, Greece, September 24-26, 2008 : proceedings". Berlín: Springer, 2008, p. 205-214.

The final authenticated version is available online at https://doi.org/10.1007/978-3-540-87781-3_23 
what they will potentially need in order to develop their future academic or professional activities [2].

The essential issue here is first how to design a CSCL setting that can be used for real, long-term, complex collaborative problem solving situations and which enables the instructor to both analyze group interaction effectively and provide an adequate support when needed. Secondly, how to extract relevant knowledge from collaboration in order to provide learners with efficient awareness and feedback as regards individual and group performance and assessment. We therefore base the success of CSCL applications on the capability of such applications to embed information and knowledge extracted from group activity interaction and use it to achieve a more effective group monitoring [3].

Large amounts of information data are generated from asynchronous interaction, usually stored in log files, which includes complex issues of the collaborative work and learning process (e.g., group well-being as well as self-, peer- and group activity evaluation [3]). This knowledge is then fed back and presented to the participants in a suitable manner to greatly influence the collaborative process by allowing students to compare their individual performance to the group one [4]. This information also serves for the instructor to identify groups with a low activity level, which allows for establishing just-in-time assistance for them [5].

The real context in this study is the virtual learning environment of the Open University of Catalonia (UOC) ${ }^{1}$. Part of UOC courses' curricula includes the participation of students in on-line discussions with the aim of sharing and discussing their ideas. Indeed, the discussion process plays an important social task where participants can think about the activity being performed, collaborate with each other through the exchange of ideas that may arise, propose new resolution mechanisms, as well as justify and refine their own contributions and thus acquire new knowledge [6].

The paper is organized as follows: Section 2 introduces the main ideas of a theoretical framework for collaborative discussion processes, whose purpose is both to model the main interactions that describe a generic discussion process and to provide a global scheme for monitoring purposes. Based on these principles, Section 3 reports on the experiences and the evaluation results achieved from using ad hoc computer systems that provide effective knowledge to students and assist instructors by means of monitoring reports on the discussion process. Finally, Section 4 concludes by summarizing the main contribution presented in this paper and outlining ongoing and future work.

\section{Use of Interaction Data Analysis in a Discussion Process}

Given the added value of asynchronous online discussion groups as one of the main elements of the pedagogical model in many educational organizations, it is essential

\footnotetext{
${ }^{1}$ The Open University of Catalonia (UOC) is located in Barcelona, Spain. The UOC offers full distance education through the Internet since 1994. About 50,000 students, lecturers and tutors are involved in 600 on-line official courses from 23 official degrees and other $\mathrm{PhD}$ and postgraduate programs. The UOC is found at http://www.uoc.edu
} 
to provide adequate on-line tools to support the whole discussion process, which also includes students' monitoring and evaluation. In this context, an important issue raised in collaborative learning interactions is the change from divergence to shared understanding and to possible construction of knowledge. The point is to understand how collaborative interactions develop over time: whether students raise new issues (ideas) more frequently as they become more familiar with the discussion and discussants, and whether shared knowledge building becomes richer over time, and subsequent evidence that students were able to construct their own understanding based on their interactions with others in formal educational settings [7], [8], [9]. To this end, our model annotates and examines a variety of elements that contribute to the understanding of the nature of the collaborative interactions, such as the students' passivity, proactivity, reactivity as well as the effectiveness and impact of their contributions to the overall goal of the discussion (see a complete description of this model in [4]).

In overall, we believe that there are more evident key discourse elements and aspects that play an important role both for promoting student participation and enhancing group and individual performance, such as, the impact and effectiveness of students' contributions, among others, that we explore in this work. By making these elements explicit, our discussion model accomplishes high students' participation rates and contribution quality in a more natural and effective way. Indeed, our approach goes beyond a mere interaction analysis of asynchronous discussion in the sense that it builds a multi-functional model that fosters knowledge sharing and construction, develops a strong sense of community among students, provides the instructor a powerful tool for students' monitoring and discussion regulation, while it allows peer facilitation through self-, peer- and group awareness and assessment. To this end, it is important that the system is capable of managing both qualitative and quantitative information and transforming it into useful knowledge for all the implicated parties in an efficient and clear way.

Furthermore, instructors need to face the important challenge of the high dropout rates typically found in any type of distance program and activity [2]. Indeed, the nature of distance education can create a sense of isolation in learners who can feel disconnected from the instructor, the rest of the class, and even the institution. It is necessary, then, that instructors provide just-in-time guidance and assistance to students' activities and also that they provide regular feedback on these activities. However, the evaluation of hundreds of contributions in a multi-member discussion and thoroughly track all the activities performed can be a tedious and time-consuming task for instructors and should be adequately supported. It is even much more complex to figure out the interactions taking place among students and/or groups of students, to identify the relevant actors -groups' leaders and followers-, to detect students that are likely to dropout the course, or to perceive possible group internal conflicts or malfunction before it gets too late to efficiently manage these problems [1], [3]. To this end, our model on interactions includes the provision of monitoring reports that can be used by instructors to easily track down the learners' online behavior and group's activity at specific milestones during the discussion process, gather feedback from the learners and scaffold groups with low degree of activity as well as to regulate the discussion by providing just-in-time assistance according to groups' and students' necessities. 
Consequently, the analysis of the interactions occurring in an online discussion process yields very useful conclusions on aspects such as individual and group working, dynamics, performance and success, which allows the instructor to obtain a global account of the progress of the individual and group work and thus to identify possible conflicts and monitor the whole learning process much better. The aim is both to provide reliable indicators that qualify the contributions and to promote the discussion's dynamics by increasing the users' interaction with the system. The definition and measurement of the indicators used to assess participation behavior, knowledge building and performance are entirely discussed in [4].

\section{A Knowledge Building and Monitoring System}

In order to validate the previous ideas, in this section, we introduce both a prototype of a web-based structured discussion forum system, called Discussion Forum (DF) (see [4] for a detailed description of this application) and a global scheme of the monitoring system called Student Activity Monitoring using Overview Spreadsheets (SAMOS) [5], which were developed to bring new opportunities to learning by discussion and to meet new pedagogical models. We report here on this novel experience and the results achieved in the real learning context of the Open University of Catalonia.

\subsection{An Effective Structured Discussion Forum}

The DF supports a complete discussion and reasoning process based on specific types of generic contributions, namely problem statement, elaboration and consensus. The problem statement occurs during the initial stage of the discussion process carried out by the instructor or group member who contributes by defining a new situation to discuss. Elaboration refers to specific contributions in which a sub-problem is stated, extended, and finally reached a consensus on a solution proposed. Finally, when one or more solutions are proposed, the consensus exchanges take place for their approval. When a solution is finally approved, the discussion terminates.

In order to support this discussion process, the DF design includes certain thematic annotation cards based on the low-level exchange categories (Fig. 1), such as information-clarification and request of opinion that qualifies each contribution and as a result structure the discussion process conveniently for later processing and analysis. Consequently, following the principles mentioned in Sect. 2, all contributions are recorded, analyzed as information and the knowledge extracted is presented to participants in (almost) real time with the aim of guiding directly students during the learning activity and also for monitoring purposes. Fig. 2 shows the provision to students of updated knowledge about the discussion in the form of complex feedback. This feedback allows students to compare their performance to the group as well as to identify which dimension of their participation needs to be improved.

Finally, the DF provides additional features to support the discussion in comparison to traditional discussion tools, such as discussion threads in fully separated rooms and open-closed branched dialogs [4]. 


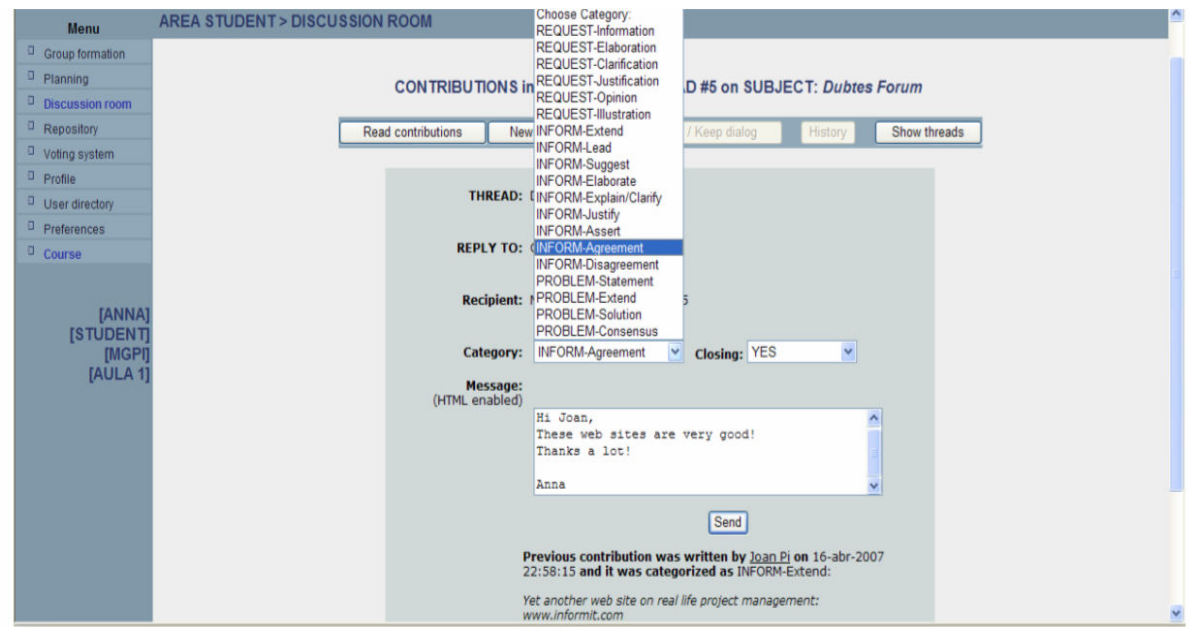

Fig. 1. A list of tags to qualify a contribution.

In order to evaluate our prototype of the DF and analyze its effects in the discussion process, 80 graduated students enrolled in the course Methodology and Management of Computer Science Projects during the last term were involved in this experience. Students were equally distributed into two classrooms and participated in the experience at the same time and during the same time (i.e., a fortnight). Students from one classroom were required to use the well-known asynchronous threaded discussion forum offered by the UOC virtual campus while the other group of students used the new DF outside the campus to support the same discussion with the same rules.

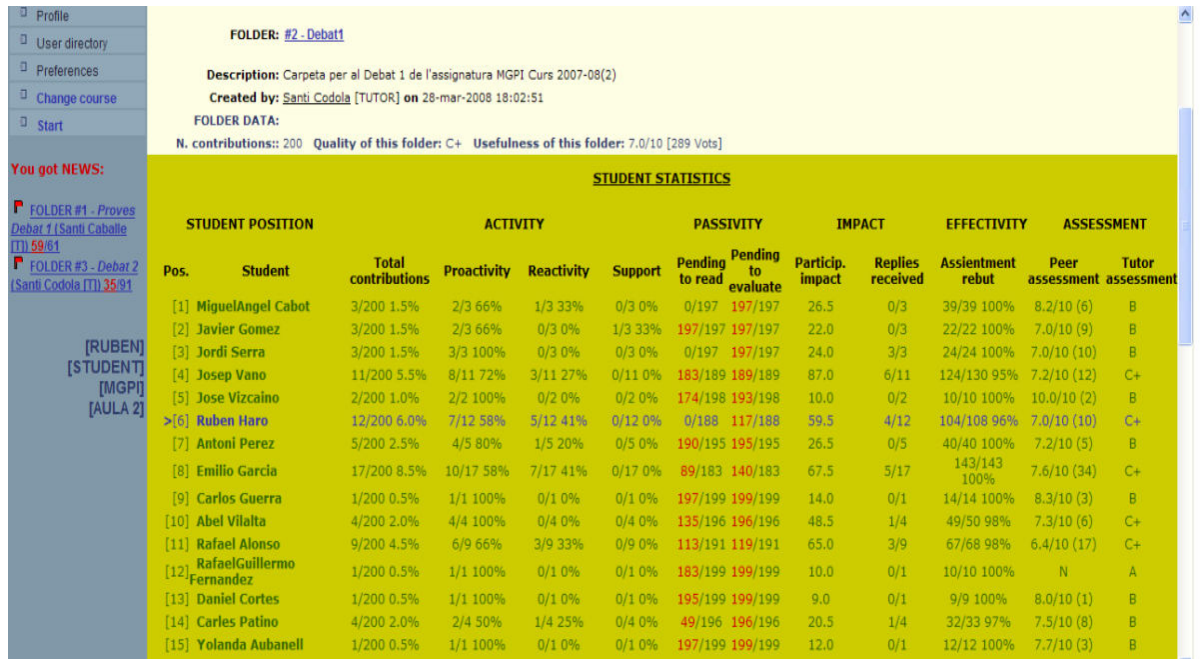

Fig. 2. A snapshot of complex and updated feedback provided to all participants. 
In comparison to the traditional discussion tool used in the other classroom, the DF provided students with relevant feedback from the discussion process, such as the current mean number of all contributions' qualitative mark for each student, peer assessment, passivity level, and the impact value and effectiveness of each student's contributions (Fig. 2).

A statistical analysis of the results comparing both the standard and the DF tools is shown in Table 1. Despite the standard tool generated more threads, most of them were actually empty (i.e. just 8 threads were contributed with more than 1 post vs. 42 threads in the DF). Moreover, the standard deviation (SD) for the posts/thread mean appears to be high in the DF, which proves the heterogeneity of the discussion involving threads of very different length. Finally, note the very high SD statistic in the posts/students mean due to a single outlier, without which SD is 6,3 .

Table 1. Main statistics extracted from the discussion using both discussion tools.

\begin{tabular}{lcc}
\hline \multicolumn{1}{c}{ Statistics } & Standard tool & Discussion Forum (DF) \\
\hline Number of students & 40 & 40 \\
\hline Number of threads & 48 & 44 \\
\hline Total of posts & 95 & 351 \\
\hline Mean number (posts/thread) & $\mathrm{M}=1.9 \mathrm{SD}=2.4$ & $\mathrm{M}=7.9 \mathrm{SD}=5.0$ \\
Mean number (posts/student) & $\mathrm{M}=2.3 \quad \mathrm{SD}=1.9$ & $\mathrm{M}=8.7 \quad \mathrm{SD}=8.1$ \\
Mean number & $\mathrm{M}=352 \quad \mathrm{SD}=139$ & $\mathrm{M}=286 \quad \mathrm{SD}=85$
\end{tabular}

The results of the semi-automatic assessment were very promising since the instructor in charge of the DF agreed with the final marks proposed by the system in more than $75 \%$ of cases. 31 out of 40 students in the DF's rank (see Fig. 2) matched the same position as in the rank appeared in the instructor's manual evaluation. In addition, the instructor reported the promising benefits from the DF in the monitoring process since this new tool alleviates instructors and moderators from the tedious work of tracking and evaluating the discussion's dynamics and outcomes manually. Next, we present additional mechanisms for the instructor to monitor the discussion.

\subsection{The Global Scheme of a Monitoring System}

This sub section shows the global scheme of the monitoring system that we have developed and tested in real collaborative learning settings. The general functioning of this system is explain next (see [5] for a complete description and Fig. 3):

1. Students participate in the collaborative spaces associated to their working group: they post or read contributions in the discussion tool, send or read emails, upload or download documents, manage folders and files, etc. Each of these activities can be considered as an event of a certain type which has been carried out by a particular student at a certain time and web space.

2. Events generated by students are registered in log files in the learning management system (a BSCW server [10] in our case, but the approach is generic and it could be any other learning management system). 
3. A specific Java application, called EICA [5], is used to automatically read and process new incoming log files and to store the extracted data into a unique persistent database in the corresponding server.

4. Database files are then processed by SAMOS application. SAMOS was specifically designed and developed to generate weekly reports which summarize group and student activity levels in a graphical manner.

5. The server automatically sends these reports to instructors by e-mail.

6. Instructors receive these reports and analyze them, looking for groups and students which seem to be "at risk", i.e.: students with low activity levels -which makes them likely to be non-participating students and possible dropout students-, and groups with low activity levels -which makes them to be possible malfunctioning groups.

7. These results are then combined and contrasted with the qualitative self-, peerand group evaluation reports which are generated by the students themselves.

8. Once the groups and students at risk have been detected, and the specific problems have been identified and classified according to whether they refer to task, group functioning or group social cohesion, instructors contact them to offer specific guidance and support towards the best development and completion of their collaborative activities.

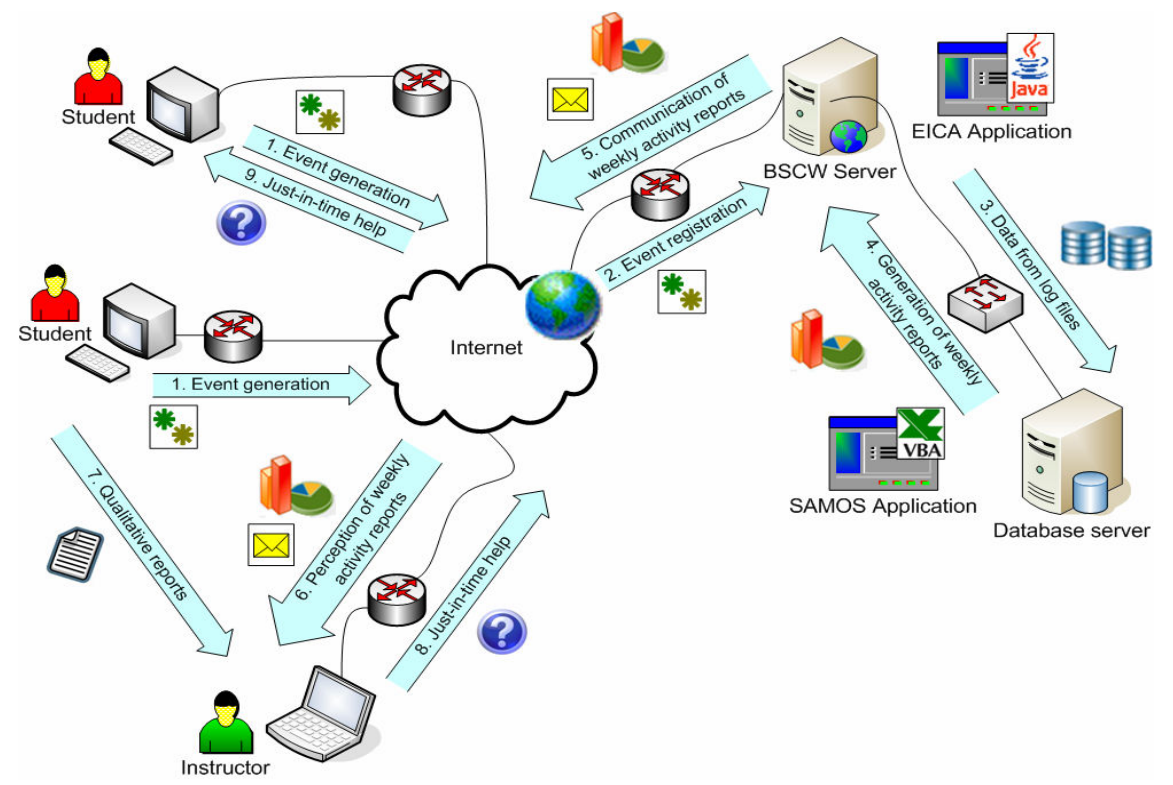

Fig. 3 General scheme of our monitoring model.

This way, students and groups at risk, receive just-in-time and efficient guidance and support so that to enhance and continue their individual or collaborative work during the discussion more successfully.

As an initial design option, we chose to generate weekly monitoring reports with the aim to show a small set of graphs that were easily and quickly understood by 
instructors, so that they did not have to invest extra time in analyzing data. To this end, we designed the following charts: (1) groups' classification graph, (2) students' classification graph, (3) group's activity-evolution graph, and (4) student's activityevolution graph.

For the sake of exemplifying the type of monitoring reports, we present here the groups' classification graph (a detailed description of the other charts can be found in [5]). This chart (Fig. 4) is a scatter plot of the following two variables: $\mathrm{X}=$ "average number of events per member that have been generated by group $i$ during this (current) week" $(i=1,2, \ldots, n)$, and $\mathrm{Y}=$ "average number of events per member that have been generated by group $i$ during an average week".

GROUPS' CLASSIFICATION BY ACTIVITY LEVEL - WEEK 6

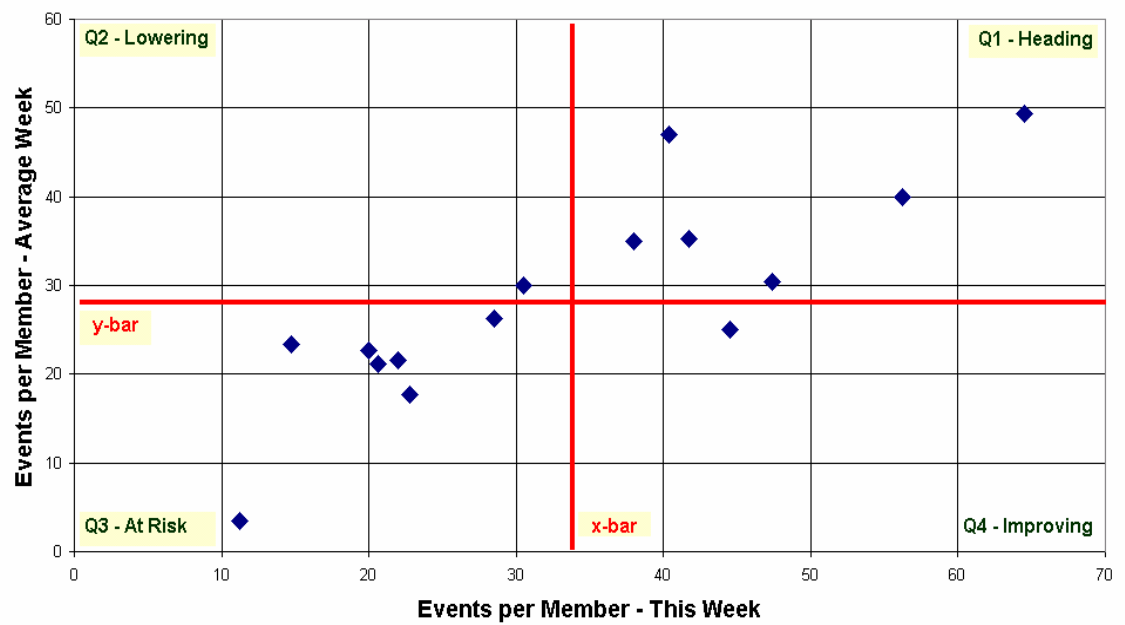

Fig. 4. Groups' classification graph.

The plot also includes the straight lines $x=\bar{x}$ and $y=\bar{y}$, which divide the graph in four quadrants, Q1 to Q4. That way, points in Q1 can be seen as representing heading groups since their activity levels are above the two activity means -current week and average week-; points in Q2 can be considered as lowering groups, since even when historically their activity level has been above the activity level for an average week, their current activity level is below the average; points in Q3 represent those groups which are below the two activity means -current and historical- and, therefore, they can be considered as groups at risk, since they are the most likely to suffer from low task contribution, group malfunctioning, lack of social cohesion and eventually from students' dropouts; finally, points in Q4 can be seen as improving groups, since even though their activity level has been historically below the mean, their level has been above the mean during the current week, so they are experimenting some improvement in their activity level. Note that these interpretations can be stronger as the distance between the considered point and the straight line is greater -e.g.: considering 
its distance from the $\mathrm{x}$-bar line, there is good evidence that the activity of the group in Q4 has been fairly improved during the current week.

In order to test whether the information provided by SAMOS may significantly influence groups' and students' performance in real collaborative learning situations in a more systematic and extensive way, we carried out the following experiment during the last academic term: a random sample of size 40 students was drawn from the population of groups that were participating in several discussions in collaborative elearning courses. During the semester, instructors of these selected groups were provided with weekly reports generated by SAMOS, so that they could detect students and groups at risk and provide them with just-in-time guidance and support. At the end of the semester, we calculated the following indexes:

1. Percentage of sampled groups which concluded their discussions according to its initial specifications (PGF).

2. Percentage of sampled groups which received a positive evaluation of their discussions at the end of the semester (PGP).

3. Percentage of sampled groups which experimented dropouts (PGD) -that is, some of the group members abandoned the discussion during the semester.

Moreover, we used historical data from previous semesters to obtain the beforeSAMOS population percentages for these indexes, $p_{0}^{i}(i=1,2,3)$. Then, for each selected index $i$, we considered the corresponding hypothesis tests about the population proportions, i.e.: $H_{0}: p_{S A M O S}^{i}=p_{0}^{i}$ versus $H_{A}: p_{S A M O S}^{i} \neq p_{0}^{i}$. Both percentages and results for these tests are shown in Table 2.

Table 2. Hypothesis tests about the population proportions.

\begin{tabular}{l|c|c|c|c}
\hline Index & $p_{0}$ (Historical) & $p_{\text {SAMOS }}(\mathrm{n}=40)$ & $95 \%$ CI & p-value \\
\hline 1. PGF & $55 \%$ & $75 \%(30)$ & $(0.59,0.87)$ & 0.011 \\
\hline 2. PGP & $49 \%$ & $65 \%(26)$ & $(0.48,0.79)$ & 0.056 \\
\hline 3. PGD & $43 \%$ & $25 \%(10)$ & $(0.13,0.41)$ & 0.025 \\
\hline
\end{tabular}

Using a standard significance level, $\alpha=0.05$, we could conclude from the corresponding p-values that the tests associated with indexes 1 and 3 were significant. In other words, statistical evidence supports the idea that the information provided by SAMOS has contributed to significantly enhance the PGF and PGD indexes in collaborative e-learning courses offered in our e-learning environment.

\section{Conclusions and Future Work}

This paper describes a promising approach for enhancing knowledge management that contributes to the improvement of the discussion process in virtual collaborative learning environments from both the knowledge building and monitoring perspective. The results of the experiences reported here are not conclusive due to its exploratory nature. However, from the analysis of the results it has been proved to promise sig- 
nificant benefits for students in the context of discussion by learning, project-based learning, and in education in general.

A decentralized distributed infrastructure has been recently added to our prototypes [11] in order to meet certain important non-functional requirements that may influence the learning process a great deal, such as performance, scalability, faulttolerance, and interoperability. The gain in performance might help us, for instance, include more complex information of the collaboration to be generated and presented in real time (such as modelling the participants' behaviour during the discussion by combining individual and group session and navigation information). We plan to explore these interesting possibilities further.

Acknowledgements. This work has been partially supported by the Spanish MCYT project TSI2005-08225-C07-05.

\section{References}

1. Dillenbourg, P. (ed.): What do you mean by "Collaborative Learning"?. Collaborative learning. Cognitive and computational approaches, 1-19. Oxford: Elsevier Science (1999)

2. Simonson, M., Smaldino, S., Albright, M., Zvacek, S.: Teaching and Learning at a Distance. Prentice Hall, Upper Saddle River, NJ. (2003)

3. Daradoumis, T., Martínez, A., Xhafa, F.: A Layered Framework for Evaluating Online Collaborative Learning Interactions. Int. J. of Human-Computer Studies, 64(7), 622-635 (2006)

4. Caballé, S., Daradoumis, Th., Xhafa, F.: Providing an Effective Structured and Contextaware Asynchronous Discussion Forum for Collaborative Knowledge Building. In proceedings of the World Conference on Educational Multimedia, Hypermedia and Telecommunications (ED-MEDIA 2008). Vienna, Austria. AACE Press (2008)

5. Juan, A., Daradoumis, T., Faulin, J., Xhafa, F.: Developing an Information System for Monitoring Student's Activity in Online Collaborative Learning. In Proceedings of the Second International Conference on Complex, Intelligent and Software Intensive Systems (CISIS'08). Barcelona, Spain. IEEE Computer Society (2008)

6. Stahl, G.: Group Cognition: Computer Support for Building Collaborative Knowledge. Acting with Technology Series. MIT Press, Cambridge, MA (2006)

7. Puntambekar, S.: Analyzing collaborative interactions: divergence, shared understanding and construction of knowledge. Computers \& Education. 47(3): 332-351, Academic Press: Elsevier Ltd. (2006)

8. Schellens, T., Valcke, M.: Fostering knowledge construction in university students through asynchronous discussion groups. Computers \& Education. 46(4):349-370, Academic Press: Elsevier Ltd. (2006)

9. Soller, A.: Supporting Social Interaction in an Intelligent Collaborative Learning System. Int. J. of Artificial Intelligence in Education, 12: 40-62 (2001)

10. Bentley, R., Appelt, W., Busbach, U., Hinrichs, E., Kerr, D., Sikkel, S., Trevor, J., Woetzel, G.: Basic Support for Cooperative Work on the World Wide Web. International Journal of Human-Computer Studies 46(6) 827-846 (1997)

11. Caballé, S., Xhafa, F., Daradoumis, T.: A Service-oriented Platform for the Enhancement and Effectiveness of the Collaborative Learning Process in Distributed Environments. LNCS 4804. (pp. 1280-1287). Heidelberg-Berlin: Springer-Verlag (2007) 